\title{
Pullback attractors for non-autonomous 2D-Navier-Stokes equations in some unbounded domains
}

\author{
T. Caraballo ${ }^{\mathrm{a}, *}$, G. Łukaszewicz ${ }^{\mathrm{b}, * *}$, and J. Real ${ }^{\mathrm{a}, *}$ \\ a Dpto. de Ecuaciones Diferenciales y Análisis Numérico, Universidad de Sevilla, Apdo. de Correos 1160, 41080 Sevilla, \\ Spain \\ ${ }^{\mathrm{b}}$ University of Warsaw, Institute of Applied Mathematics and Mechanics, Banacha 2, 02-097 Warsaw, Poland
}

\begin{abstract}
In this Note we first introduce the concept of pullback asymptotic compactness. Next, we establish a result ensuring the existence of a pullback attractor for a non-autonomous dynamical system under the general assumptions of pullback asymptotic compactness and the existence of a pullback absorbing family of sets. Finally, we prove the existence of a pullback attractor for a non-autonomous 2D Navier-Stokes model in an unbounded domain, a case in which the theory of uniform attractors does not work since the non-autonomous term is quite general.
\end{abstract}

\section{Résumé}

Dans cette Note, on présente d'abord la notion de compacité asymptotique pullback. On établit ensuite un résultat d'existence d'un attracteur pullback pour un système dynamique non autonome, sous les hypothèses de compacité asymptotique pullback et d'existence d'une famille d'ensembles absorbants au sens pullback. On prouve finalement l'existence d'un attracteur pullback pour un système de Navier-Stokes bidimensionel non autonome dans un domaine non borné, une situation dans laquelle, étant donnée la généralité du terme non autonome, la théorie des attracteurs uniformes ne peut pas être appliquée.

\section{Version française abrégée}

La numérotation, les notations et la terminologie que nous utilisons se réfèrent directement à la numérotation, aux notations et à la terminologie de la version anglaise.

On appelle processus sur un espace métrique $X$ toute famille $\{U(t, \tau) ;-\infty<\tau \leq t<+\infty\}$ d'applications continues $U(t, \tau): X \rightarrow X$, telle que $U(\tau, \tau) x=x$, et (3) est satisfaite. On suppose donnée une famille non vide $\mathcal{D}$ d'ensembles $\widehat{D}=\{D(t) ; t \in \mathbb{R}\} \subset \mathcal{P}(X)$, où on dénote par $\mathcal{P}(X)$ la classe de tous les

\footnotetext{
* Supported by Ministerio de Ciencia y Tecnología (Spain) and FEDER (European Community) grant BFM2002-03068

**Supported by Polish Government grant KBN 2 P303A 03022

Email addresses: caraball@us.es (T. Caraballo), glukasz@mimuw.edu.pl (G. Lukaszewicz), jreal@us.es (J. Real).
} 
sous-ensembles non vides de $X$. On introduit les notions de processus $\mathcal{D}$-asymptotiquement compact au sens pullback (Definition 1), de famille $\mathcal{D}$-absorbante pullback (Definition 2) et de $\mathcal{D}$-attracteur pullback (Definition 3). Notre résultat principal est le suivant :

Théorème 0.1. Supposons que le processus $U(\cdot, \cdot)$ est $\mathcal{D}$-asymptotiquement compact au sens pullback, et que $\widehat{B} \in \mathcal{D}$ est une famille $\mathcal{D}$-absorbante pullback. Alors, la famille $\widehat{A}=\{A(t) ; t \in \mathbb{R}\} \subset \mathcal{P}(X)$ definie par $A(t)=\Lambda(\widehat{B}, t), t \in \mathbb{R}$, où pour chaque $\widehat{D} \in \mathcal{D}$

$$
\Lambda(\widehat{D}, t)=\bigcap_{s \leq t}\left(\overline{\bigcup_{\tau \leq s} U(t, \tau) D(\tau)}\right),
$$

est un D-attracteur pullback pour $U(\cdot, \cdot)$, et satisfait que $A(t)=\overline{\bigcup_{\widehat{D} \in \mathcal{D}} \Lambda(\widehat{D}, t)}$, pour tout $t \in \mathbb{R}$. De plus, $\widehat{A}$ est minimal au sens que si $\widehat{C}=\{C(t) ; t \in \mathbb{R}\} \subset \mathcal{P}(X)$ est une famille d'ensembles fermés telle que $\lim _{\tau \rightarrow-\infty} \operatorname{dist}(U(t, \tau) B(\tau), C(t))=0$, alors $A(t) \subset C(t)$.

On applique le Théorème 0.1 au système de Navier-Stokes bidimensionel non autonome (2) dans un domaine non borné satisfaisant (1). On denote $\sigma=\nu \lambda_{1}$, et $\mathcal{R}_{\sigma}$ l'ensemble de toutes les fonctions $r: \mathbb{R} \rightarrow$ $(0,+\infty)$ qui satisfont $(6)$. On definit $\mathcal{D}_{\sigma}$ comme étant la classe de toutes les familles $\widehat{D}=\{D(t) ; t \in$ $\mathbb{R}\} \subset \mathcal{P}(H)$ pour lesquelles il y a une fonction $r_{\widehat{D}} \in \mathcal{R}_{\sigma}$ telle que $D(t) \subset \bar{B}\left(0, r_{\widehat{D}}(t)\right)$, et on démontre le résultat suivant :

Théorème 0.2. Supposons que $f \in L_{\text {loc }}^{2}\left(\mathbb{R} ; V^{\prime}\right)$ et satisfait $\int_{-\infty}^{t} e^{\sigma \xi}\|f(\xi)\|^{2} d \xi<+\infty$ pour tout $t \in \mathbb{R}$. Alors, il existe un unique $\mathcal{D}_{\sigma}$-attracteur pullback, appartenant $\grave{a} \mathcal{D}_{\sigma}$, pour le processus $U(\cdot, \cdot)$ defini par (4).

\section{Introduction}

Let $\mathcal{O} \subset \mathbb{R}^{2}$ be an open set, not necessarily bounded, with boundary $\partial \mathcal{O}$, and suppose that $\mathcal{O}$ satisfies the Poincaré inequality, i.e., there exists a constant $\lambda_{1}>0$ such that

$$
\lambda_{1} \int_{\mathcal{O}} \phi^{2} d x \leq \int_{\mathcal{O}}|\nabla \phi|^{2} d x \quad \text { for all } \phi \in H_{0}^{1}(\mathcal{O}) .
$$

Consider the following 2D-Navier-Stokes problem (for further details see Lions [8] and Temam [14]):

$$
\left\{\begin{array}{l}
\frac{\partial u}{\partial t}-\nu \Delta u+\sum_{i=1}^{2} u_{i} \frac{\partial u}{\partial x_{i}}=f(t)-\nabla p \quad \text { in }(\tau,+\infty) \times \mathcal{O} \\
\operatorname{div} u=0 \text { in }(\tau,+\infty) \times \mathcal{O} \\
u=0 \text { on }(\tau,+\infty) \times \partial \mathcal{O} \\
u(\tau, x)=u_{0}(x), \quad x \in \mathcal{O}
\end{array}\right.
$$

This problem is set in a suitable abstract framework by considering the usual abstract spaces:

$$
\mathcal{V}=\left\{u \in\left(C_{0}^{\infty}(\mathcal{O})\right)^{2} ; \operatorname{div} u=0\right\},
$$


$H=$ the closure of $\mathcal{V}$ in $\left(L^{2}(\mathcal{O})\right)^{2}$ with norm $|\cdot|$, and inner product $(\cdot, \cdot)$ where

$$
(u, v)=\sum_{j=1}^{2} \int_{\mathcal{O}} u_{j}(x) v_{j}(x) d x, \text { for } u, v \in\left(L^{2}(\mathcal{O})\right)^{2} .
$$

$V=$ the closure of $\mathcal{V}$ in $\left(H_{0}^{1}(\mathcal{O})\right)^{2}$ with norm $\|\cdot\|$, and associated scalar product $((\cdot, \cdot))$, where

$$
((u, v))=\sum_{i, j=1}^{2} \int_{\mathcal{O}} \frac{\partial u_{j}}{\partial x_{i}} \frac{\partial v_{j}}{\partial x_{i}} d x, \text { for } u, v \in\left(H_{0}^{1}(\mathcal{O})\right)^{2} .
$$

It follows that $V \subset H \equiv H^{\prime} \subset V^{\prime}$, where the injections are continuous and dense.

Finally, we will use $\|\cdot\|_{*}$ for the norm in $V^{\prime}$ and $\langle\cdot, \cdot\rangle$ for the duality pairing between $V$ and $V^{\prime}$.

Consider the trilinear form $b$ on $V \times V \times V$ given by

$$
b(u, v, w)=\sum_{i, j=1}^{2} \int_{\mathcal{O}} u_{i} \frac{\partial v_{j}}{\partial x_{i}} w_{j} d x, \quad u, v, w \in V,
$$

define $B: V \times V \rightarrow V^{\prime}$ by $\langle B(u, v), w\rangle=b(u, v, w)$, for $u, v, w \in V$, and denote $B(u)=B(u, u)$. Assume now that $u_{0} \in H, f \in L_{l o c}^{2}\left(\mathbb{R} ; V^{\prime}\right)$. For each $\tau \in \mathbb{R}$ we consider the problem:

$$
\left\{\begin{array}{l}
u \in L^{2}(\tau, T ; V) \cap L^{\infty}(\tau, T ; H) \quad \text { for all } T>\tau, \\
\frac{\mathrm{d}}{\mathrm{d} t}(u(t), v)+\nu((u(t), v))+\langle B(u(t)), v\rangle=\langle f(t), v\rangle, \quad \text { for all } v \in V, \\
\text { in the sense of scalar distributions on }(\tau,+\infty), \\
u(\tau)=u_{0} .
\end{array}\right.
$$

It follows [14] that problem (2) has a unique solution, $u\left(\cdot ; \tau, u_{0}\right)$, that moreover belongs to $C^{0}([\tau,+\infty) ; H)$.

It is known that the theory of global attractors previously developed for autonomous systems is no longer valid to analyse the long-term behaviour of this kind of problems, and it is necessary to use some techniques for non-autonomous systems. Having several possible options (e.g. kernel sections [4], skewproduct semiflows [13], etc..), we will use the already well established theory of pullback attractors (Crauel et al. [5], Langa and Schmalfuss [7], Kloeden and Schmalfuss [6], Schmalfuss [12]) since it allows to handle more general non-autonomous terms, and it works under the presence of random environments as well.

In order to prove the existence of the attractor we will exploit an approach which is based on the use of the energy equations which are in direct connection with the concept of asymptotic compactness, and which permits us to obtain informations on the asymptotic behaviour of our systems when the injection $V \hookrightarrow H$ is not compact (as in our case of unbounded domain). This method was first used by Ball (see e.g. [1]) in the context of autonomous dynamical systems, and by Lukaszewicz and Sadowski in [9] (and later also in [10]) to extend to the non-autonomous situation the corresponding one in the autonomous framework (see Rosa [11]), but related to uniform asymptotic compactness. Now we are interested in the case without uniformity properties.

In Section 2 we introduce the concept of asymptotically compact non-autonomous dynamical system, and establish a general result ensuring the existence of a minimal pullback attractor under assumptions of asymptotic compactness and existence of a family of absorbing sets. Then, we prove the existence of a pullback attractor for our 2D Navier-Stokes model in an unbounded domain in which the external force needs not be bounded, neither almost periodic nor translation compact. It is enough that this term satisfies an appropriate integrability condition. 


\section{Pullback attractors for asymptotically compact non-autonomous dynamical systems}

Although it is possible to state all our analysis using the cocycle formalism (see [3]), which has proven also very useful, in particular, in the case of random dynamical systems, we prefer to use the language of evolutionary processes since it seems to be more appropriate for our situation.

Let us consider a process (also called a two-parameter semigroup) $U$ on a metric space $X$, i.e., a family $\{U(t, \tau) ;-\infty<\tau \leq t<+\infty\}$ of continuous mappings $U(t, \tau): X \rightarrow X$, such that $U(\tau, \tau) x=x$, and

$$
U(t, \tau)=U(t, r) U(r, \tau) \quad \text { for all } \tau \leq r \leq t .
$$

Suppose $\mathcal{D}$ is a nonempty class of parameterized sets $\widehat{D}=\{D(t) ; t \in \mathbb{R}\} \subset \mathcal{P}(X)$, where $\mathcal{P}(X)$ denotes the family of all nonempty subsets of $X$.

Definition 1 The process $U(\cdot, \cdot)$ is said to be pullback $\mathcal{D}$-asymptotically compact if for any $t \in \mathbb{R}$, any $\widehat{D} \in \mathcal{D}$, any sequence $\tau_{n} \rightarrow-\infty$, and any sequence $x_{n} \in D\left(\tau_{n}\right)$, the sequence $\left\{U\left(t, \tau_{n}\right) x_{n}\right\}$ is relatively compact in $X$.

Definition 2 It is said that $\widehat{B} \in \mathcal{D}$ is pullback $\mathcal{D}$-absorbing for the process $U(\cdot, \cdot)$ if for any $t \in \mathbb{R}$ and any $\widehat{D} \in \mathcal{D}$, there exists a $\tau_{0}(t, \widehat{D}) \leq t$ such that

$$
U(t, \tau) D(\tau) \subset B(t) \quad \text { for all } \tau \leq \tau_{0}(t, \widehat{D}) .
$$

Definition 3 The family $\widehat{A}=\{A(t) ; t \in \mathbb{R}\} \subset \mathcal{P}(X)$ is said to be a pullback $\mathcal{D}$-attractor for $U(\cdot, \cdot)$ if

(i) $A(t)$ is compact for all $t \in \mathbb{R}$,

(ii) $\widehat{A}$ is pullback $\mathcal{D}$-attracting, i.e.,

$$
\lim _{\tau \rightarrow-\infty} \operatorname{dist}(U(t, \tau) D(\tau), A(t))=0 \quad \text { for all } \widehat{D} \in \mathcal{D}, \text { and all } t \in \mathbb{R},
$$

(iii) $\widehat{A}$ is invariant, i.e.,

$$
U(t, \tau) A(\tau)=A(t), \quad \text { for }-\infty<\tau \leq t<+\infty .
$$

Remark 4 Observe that Definition 3 does not guarantee the uniqueness of pullback $\mathcal{D}$-attractors (see Caraballo and Langa [2] for a discussion on this point). In order to ensure uniqueness one needs to impose additional conditions as, for instance, the condition that the attractor belongs to the same family $\mathcal{D}$, or some kind of minimality. However, we do not include this stronger assumptions in the definition since, as we will show in Theorem 5, under very general hypotheses, it is possible to ensure the existence of a global pullback $\mathcal{D}$-attractor which is minimal in an appropriate sense.

We have the following result

Theorem 5 Suppose that the process $U(\cdot, \cdot)$ is pullback $\mathcal{D}$-asymptotically compact and that $\widehat{B} \in \mathcal{D}$ is a family of pullback $\mathcal{D}$-absorbing sets for $U(\cdot, \cdot)$.

Then, the family $\widehat{A}=\{A(t) ; t \in \mathbb{R}\} \subset \mathcal{P}(X)$ defined by $A(t)=\Lambda(\widehat{B}, t), \quad t \in \mathbb{R}$, where for each $\widehat{D} \in \mathcal{D}$

$$
\Lambda(\widehat{D}, t)=\bigcap_{s \leq t}\left(\overline{\bigcup_{\tau \leq s} U(t, \tau) D(\tau)}\right),
$$

is a pullback $\mathcal{D}$-attractor for $U(\cdot, \cdot)$ which satisfies in addition that $A(t)=\overline{\bigcup_{\widehat{D} \in \mathcal{D}} \Lambda(\widehat{D}, t)}$, for $t \in \mathbb{R}$. Furthemore, $\widehat{A}$ is minimal in the sense that if $\widehat{C}=\{C(t) ; t \in \mathbb{R}\} \subset \mathcal{P}(X)$ is a family of closed sets such that $\lim _{\tau \rightarrow-\infty} \operatorname{dist}(U(t, \tau) B(\tau), C(t))=0$, then $A(t) \subset C(t)$. 
PROOF. (Sketch) The proof follows from the properties of the omega limit sets $\Lambda(\widehat{D}, t)$. Indeed, it follows that $\Lambda(\widehat{D}, t)$ is a compact nonempty set for all $\widehat{D} \in \mathcal{D}$ and all $t \in \mathbb{R}$. Moreover, the family $\{\Lambda(\widehat{D}, t), t \in \mathbb{R}\}$ pullback attracts $\widehat{D}$, is invariant in the sense of Definition 3 , and satisfies that $\Lambda(\widehat{B}, t)=$ $\overline{\bigcup_{\widehat{D} \in \mathcal{D}} \Lambda(\widehat{D}, t)}$. The minimality property follows immediately (see [3] for more details).

Remark 6 The family $\widehat{A}$ is said to be forward attracting if

$$
\lim _{t \rightarrow+\infty} \operatorname{dist}(U(t, \tau) D(\tau), A(t))=0 \quad \text { for all } \widehat{D} \in \mathcal{D} \text {, and all } \tau \in \mathbb{R} .
$$

It is interesting to investigate when a pullback attractor also attracts in the forward sense. In this respect, if the pullback attraction is uniform, i.e. if it holds

$$
\lim _{s \rightarrow+\infty} \sup _{t \in \mathbb{R}} \operatorname{dist}(U(t, t-s) D(t-s), A(t))=0 \quad \text { for all } \widehat{D} \in \mathcal{D},
$$

then, equivalently, it also holds the uniform forward attraction property

$$
\lim _{t \rightarrow+\infty} \sup _{\tau \in \mathbb{R}} \operatorname{dist}(U(t+\tau, \tau) D(\tau), A(t+\tau))=0 \quad \text { for all } \widehat{D} \in \mathcal{D} .
$$

As far as we know, apart from the result by Chepyzhov and Vishik [4], which ensures existence of a uniform forward (and so pullback) attractor when the universe $\mathcal{D}$ is formed by fixed bounded sets and there exists a fixed compact set uniformly forward absorbing, this is still an open problem in the non-uniform case.

\section{Application to non-autonomous $2 D$-Navier-Stokes equations in unbounded domains}

Let us now apply the theory to our model. To construct the associated evolutionary process to (2), let us consider the unique solution, $u\left(\cdot ; \tau, u_{0}\right)$ to $(2)$.

Define

$$
U(t, \tau) u_{0}=u\left(t ; \tau, u_{0}\right) \quad \tau \leq t, \quad u_{0} \in H .
$$

From the uniqueness of solution to problem (2), it follows that

$$
U(t, \tau) u_{0}=U(t, r) U(r, \tau) u_{0}, \quad \text { for all } \tau \leq r \leq t, u_{0} \in H .
$$

Also, it is a standard task to prove that for all $\tau \leq t$, the mapping $U(\tau, t): H \rightarrow H$ defined by (4), is continuous. Consequently, the family $\{U(t, \tau), \tau \leq t\}$ defined by (4) is a process $U(\cdot, \cdot)$ in $H$.

From now on, we denote $\sigma=\nu \lambda_{1}$. Let $\mathcal{R}_{\sigma}$ be the set of all functions $r: \mathbb{R} \rightarrow(0,+\infty)$ such that

$$
\lim _{t \rightarrow-\infty} e^{\sigma t} r^{2}(t)=0
$$

and denote by $\mathcal{D}_{\sigma}$ the class of all families $\widehat{D}=\{D(t) ; t \in \mathbb{R}\} \subset \mathcal{P}(H)$ such that $D(t) \subset \bar{B}\left(0, r_{\widehat{D}}(t)\right)$, for some $r_{\widehat{D}} \in \mathcal{R}_{\sigma}$, where $\bar{B}\left(0, r_{\widehat{D}}(t)\right)$ denotes the closed ball in $H$ centered at zero with radius $r_{\widehat{D}}(t)$.

Now, we have

Theorem 7 Suppose that $f \in L_{l o c}^{2}\left(\mathbb{R} ; V^{\prime}\right)$ is such that $\int_{-\infty}^{t} e^{\sigma \xi}\|f(\xi)\|_{*}^{2} d \xi<+\infty$ for all $t \in \mathbb{R}$. Then, there exists a unique global pullback $\mathcal{D}_{\sigma}$-attractor belonging to $\mathcal{D}_{\sigma}$ for the process $U$ defined by (4).

PROOF. (Sketch) Let $\tau \in \mathbb{R}$ and $u_{0} \in H$ be fixed, and denote $u(t)=u\left(t ; \tau, u_{0}\right)=U(t, \tau) u_{0}$, for all $t \geq \tau$. It easily follows

$$
\frac{\mathrm{d}}{\mathrm{dt}}\left(e^{\sigma t}|u(t)|^{2}\right)+2 \nu e^{\sigma t}\|u(t)\|^{2}=\sigma e^{\sigma t}|u(t)|^{2}+2 e^{\sigma t}\langle f(t), u(t)\rangle,
$$


and

$$
e^{\sigma t}|u(t)|^{2} \leq e^{\sigma \tau}\left|u_{0}\right|^{2}+\frac{1}{\nu} \int_{\tau}^{t} e^{\sigma \xi}\|f(\xi)\|_{*}^{2} d \xi \quad \text { for all } \tau \leq t .
$$

Let $\widehat{D} \in \mathcal{D}_{\sigma}$ be given. From (8), we obtain for all $u_{0} \in D(\tau)$, and all $t \geq \tau$ that

$$
\left|U(t, \tau) u_{0}\right|^{2} \leq e^{-\sigma(t-\tau)} r_{\widehat{D}}^{2}(\tau)+\frac{e^{-\sigma t}}{\nu} \int_{-\infty}^{t} e^{\sigma \xi}\|f(\xi)\|_{*}^{2} d \xi
$$

For each $t \in \mathbb{R}$, denote by $R_{\sigma}(t)$ the constant given by $\left(R_{\sigma}(t)\right)^{2}=\frac{2 e^{-\sigma t}}{\nu} \int_{-\infty}^{t} e^{\sigma \xi}\|f(\xi)\|_{*}^{2} d \xi$, and consider the family $\widehat{B}_{\sigma}$ of closed balls in $H$ defined by $B_{\sigma}(t)=\left\{v \in H ;|v| \leq R_{\sigma}(t)\right\}$. It is straightforward to check that $\widehat{B}_{\sigma} \in \mathcal{D}_{\sigma}$, and that $\widehat{B}_{\sigma}$ is pullback $\mathcal{D}_{\sigma}$-absorbing for the process $U(\cdot, \cdot)$.

Let $\widehat{D} \in \mathcal{D}_{\sigma}$, a sequence $\tau_{n} \rightarrow-\infty$, a sequence $u_{0_{n}} \in D\left(\tau_{n}\right)$ and $t \in \mathbb{R}$, be fixed. To prove that from the sequence $\left\{U\left(t, \tau_{n}\right) u_{0_{n}}\right\}$ we can extract a subsequence that converges in $H$ we follow a scheme similar to that one in [9] but with necessary changes.

\section{References}

[1] J.M. Ball, Global attractors for damped semilinear wave equations, Discrete and Continuous Dynamical Systems 10(2004), no. 1-2, 31-52.

[2] T. Caraballo \& J.A. Langa, On the upper semicontinuity of cocycle attractors for nonautonomous and random dynamical systems, Dynamics of Continuous Discrete and Impulsive Systems A, 10(2003), 491-514.

[3] T. Caraballo, G. Łukaszewicz \& J. Real, Pullback attractors for asymptotically compact non-autonomous dynamical systems, manuscript, Technical Report no. 141 of the Institute of Applied Mathematics and Mechanics, University of Warsaw.

[4] V.V. Chepyzhov \& M.I. Vishik, Attractors for Equations of Mathematical Physics, Colloquium publications fol. 49, American Mathematical Society, Providence, Rhode Island, (2002).

[5] H. Crauel, A. Debussche \& F. Flandoli, Random attractors, J. Dyn. Diff. Eq. 9(1995), No. 2, 307-341.

[6] P.E. Kloeden \& B. Schmalfuss, Asymptotic behaviour of nonautonomous difference inclusions. Systems Control Lett. 33(1998), no. 4, 275-280.

[7] J.A. Langa \& B. Schmalfuss, Finite dimensionality of attractors for non-autonomous dynamical systems given by partial differential equations, Stochastics and Dynamics 4(2004), no. 3, 385-404.

[8] J.L. Lions, Quelques méthodes de résolution des problèmes aux limites non linéaires, Paris; Dunod, Gauthier-Villars, (1969).

[9] G. Łukaszewicz \& W. Sadowski, Uniform attractor for 2D magneto-micropolar fluid flow in some unbounded domains, Z. angew. Math. Phys. 55(2004), 1-11.

[10] I. Moise, R. Rosa \& X. Wang, Attractors for noncompact nonautonomous systems via energy equations, Discrete and Continuous Dynamical Systems 10(2004), No. 1 \& 2, 473-496.

[11] R. Rosa, The global attractor for the $2 D$ Navier-Stokes flow on some unbounded domains, Nonlinear Analysis, TMA 32(1998), No. 1, 71-85.

[12] B. Schmalfuss, Attractors for non-autonomous dynamical systems, in Proc. Equadiff 99, Berlin, Eds. B. Fiedler, K. Gröger and J. Sprekels (World Scientific, 2000), pp. 684-689.

[13] G.R. Sell, Non-autonomous differential equations and topological dynamics, I, II Trans. Amer. Math. Soc. 127(1967), 241-262, 263-283.

[14] R. Temam, Navier-Stokes equations, Theory and Numerical Analysis, 2nd. ed., North Holland, Amsterdam, (1979). 